This document is the Accepted Manuscript version of a Published Work that appeared in final form in Journal of the American Chemical Society, copyright (c) American Chemical Society after peer review and technical editing by the publisher. To access the final edited and published work see: https://dx.doi.org/10.1021/jacs.9b10403. 


\title{
Phase Transfer of Rhodium(II)-Based Metal-Organic Polyhedra Bearing Coordinatively Bound Cargo Enables Molecular Separation
}

T. Grancha, A. Carné-Sánchez, ${ }^{\star}$ L. Hernández-López, J. Albalad, I. Imaz, J. Juanhuix, D. Maspoch*

\begin{abstract}
The transfer of nanoparticles between immiscible phases can be driven by externally-triggered changes in their surface composition. Interestingly, phase transfers can enhance the processing of nanoparticles and enable their use as vehicles for transporting molecular cargo. Herein we report extension of such phase transfers to encompass porous Metal-Organic Polyhedra (MOPs). We report that a hydroxyl-functionalized, cuboctahedral Rh(II)-based MOP can be transferred between immiscible phases by $\mathrm{pH}$ changes or by cation-exchange reactions. We demonstrate use of this MOP to transport coordinatively-bound cargo between immiscible layers, including into solvents in which the cargo is insoluble. As proof-of-concept that our phase transfer approach could be used in chemical separation, we employed Rh(II)-based MOPs to separate a challenging mixture of structurally similar cyclic aliphatic (tetrahydrothiophene) and aromatic (thiophene) compounds. We anticipate that transport of coordinatively-bound molecules will open new avenues for molecular separation based on the relative coordination affinity that the molecules have for the $\mathrm{Rh}$ (II) sites of MOP.
\end{abstract}

\section{Introduction}

Phase transfer has gained importance in the synthesis, functionalization, and application of nanomaterials, especially those of inorganic nanoparticles (NPs).(1) Many applications of NPs demand transfer of as-synthesized NPs from polar to nonpolar solvents and vice versa. For example, biomedical applications demand water soluble NPs,(2) whereas processing of NPs for optoelectronic applications often involves nonpolar organic solvents.(3) These phase transfers are typically achieved by selective modulation of the hydrophilicity/hydrophobicity of NPs upon in situ modification of their surface composition. Such modifications can be driven by external stimuli, including changes in $\mathrm{pH},(4)$ light,(5) ionic strength(6) or temperature,(7) electrostatic interactions,(8) or ligand exchange reactions.(9)In situ tuning of solubility via external triggers has been recently extended to cationic metal-organic $(10,11)$ and organic cages,(12) whose phase transfer is usually driven by counteranion exchange. Given the rich host-guest chemistry of such cages, their transfer between phases has shown promise as a method to transport cargo between two immiscible phases.

Herein we report the phase transfer of metal-organic polyhedra (MOPs),(13-19) a neutral class of metal-organic cages that arise from the self-assembly of metal ions or clusters linked through carboxylate organic ligands and that exhibit permanent porosity between organic and aqueous media and vice versa. To this end, we selected the cuboctahedral Rh(II)-based MOP of formula [Rh2(OH-bdc)2(H2O)(DMA)]12 (hereafter called OHRhMOP, where $\mathrm{OH}$-bdc $=5$ hydroxy-1,3-benzenedicarboxylate and DMA = N,N-dimethylacetamide),(20) whose outer surface is functionalized with 24 hydroxyl groups (Figure 1a). We discovered that the reversible and quantitative protonation/deprotonation of the peripheral hydroxyl groups can be triggered by changing the $\mathrm{pH}$, which induces a change in solubility that enables transfer of the $\mathrm{Rh}(\mathrm{II})-\mathrm{MOP}$ between immiscible phases. Further, we found that once the anionic Rh(II)-MOP is generated, a second reversible phase transfer can occur, via cation exchange. We anticipated that these two phase transfer phenomena could be sequentially coupled via a triphasic solvent system, in which spontaneous phase transfer triggered by cation exchange would drive the (normally nonspontaneous) $\mathrm{pH}$-mediated phase transfer. Finally, we combined the reversible phase transfer of the MOP with the coordination capabilities of its $12 \mathrm{Rh}-\mathrm{Rh}$ paddlewheel units $(21,22)$ to transport coordinatively bound cargo between immiscible liquids, including into liquids in which the cargo is not soluble. As proof-of-concept, we combined the selective coordination chemistry of the $\mathrm{Rh}$ (II) sites to the phase transfer phenomena of $\mathrm{Rh}$ (II)-MOPs to separate a challenging 
mixture of a cyclic aliphatic molecule (tetrahydrothiophene) and its corresponding aromatic analogue (thiophene). Rh(II)-MOPs can thus behave as a kind of "molecular nanoparticle" combining the properties of NPs (e.g., high density of surface functionalization) and molecules (e.g., stoichiometric transformation on well-located reactive sites and solubility), opening up novel avenues for molecular transport and separation.

\section{Results and Discussion}

\section{pH-Triggered Phase Transfer}

We began our study by transferring OHRhMOP from an organic phase to an aqueous phase using a simple acid/base reaction (Figure $1 \mathrm{~b}$; SI, Video S1). To this end, we prepared a biphasic system of a solution of OHRhMOP in 1-butanol and an aqueous solution containing $\mathrm{NaOH}$. The phase transfer was partial when left spontaneously to occur (SI, Figure S1), whereas shaking the biphasic system for $5 \mathrm{~s}$ induced a complete transfer of the OHRhMOP to the aqueous phase. Since OHRhMOP contains 24 hydroxyl groups that can be deprotonated by base, we studied the transfer efficiency (TE) in function of the molar equivalents (molar equiv) of $\mathrm{NaOH}$ added to the aqueous phase. The TE was quantified by means of ultraviolet-visible spectroscopy (UV-vis) measurements on the aqueous phase after phase transfer (SI, Figures S1-S3). Using 24 molar equiv of $\mathrm{NaOH}$ in the aqueous phase (which gave a $\mathrm{pH}$ of ca. 11.5) provided a TE of $100 \%$, whereas use of fewer than 24 molar equiv gave only partial transfer. These results corroborated a phase transfer mechanism whereby quantitative deprotonation of the hydroxyl groups renders

a)
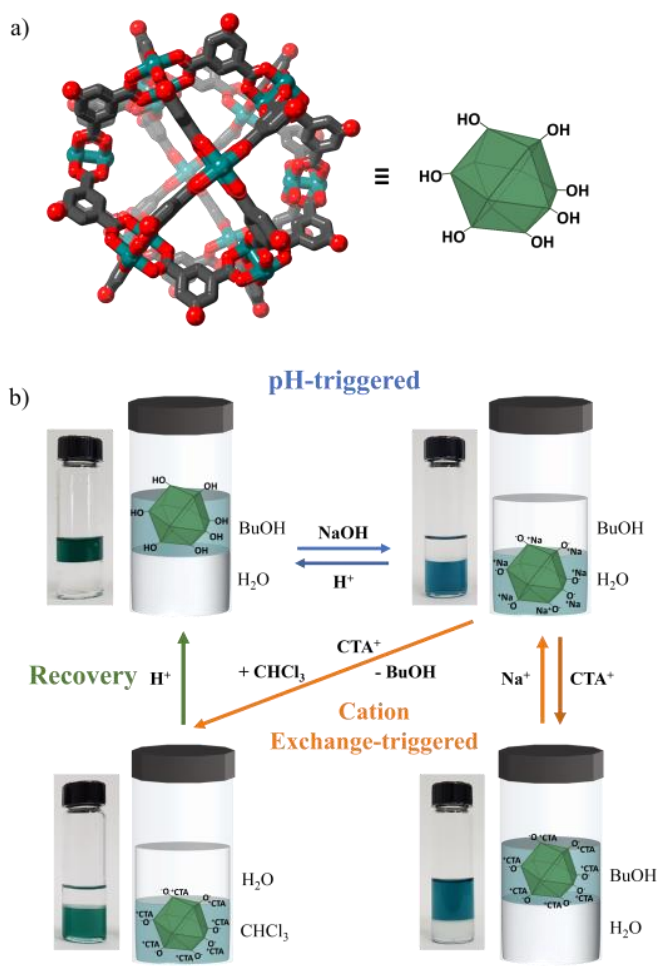

Figure 1. Crystalline structure of OHRhMOP.(20) Color key: green, rhodium; red, oxygen; gray, carbon. Hydrogen atoms and solvent molecules have been omitted for clarity. (b) Schematic representation of $\mathrm{Rh}$ (II)-MOPs during $\mathrm{pH}$ changetriggered (blue) or cation exchange-triggered (orange) phase transfers and upon recovery after precipitation with diethyl ether and subsequent dissolution in 1-butanol (green). The photographs illustrate the biphasic systems of the corresponding phase transfers. a fully deprotonated, water soluble $\mathrm{Rh}(\mathrm{II})$ MOP of formula Na24[Rh2(O-bdc)2]12 (hereafter named ONaRhMOP). The formation of ONaRhMOP was confirmed by $1 \mathrm{H} \quad \mathrm{NMR}$, energy dispersive X-ray spectroscopy (EDX), and inductively coupled plasma optical emission spectrometry (ICPOES) (SI, Figures S4, S5 and Table S1).

Next, we explored the reversibility of the phase transfer, observing complete reversibility upon addition of trifluoroacetic acid (TFA) to the 1-butanol phase (Figure 1b). We determined that 36 molar equiv of TFA are necessary to complete the transfer from water to 1-butanol, and 36 molar equiv of $\mathrm{NaOH}$ are necessary to move the Rh(II)-MOP back into water. The reversible transfer could be repeated up to 10 cycles with an overall TE of $78 \%$, estimated by comparing the maximum absorbance value in the UV-vis spectra of OHRhMOP in the 1-butanol phase before the first transfer had begun and after the 10 cycles had been completed (SI, Figures S6, S7 and Table S2). These results confirmed that $\mathrm{pH}$ changes enable phase transfer through formation of (neutral) OHRhMOP or (anionic) ONaRhMOP. The integrity of these Rh(II)MOPs under the above-mentioned basic and acidic conditions was confirmed $24 \mathrm{~h}$ after each phase transfer through UV-vis, dynamic light scattering (DLS), and matrix assisted laser desorption/ionization (time-of-flight analyzer) mass spectroscopy (MALDI-TOF) (SI, Figures S8-S10). 


\section{Cation Exchange-Triggered Phase Transfer}

Having demonstrated that reversible phase transfer of Rh(II)-MOP between 1-butanol and water could be achieved by changing the $\mathrm{pH}$, we then evaluated whether phase transfer of ONaRhMOP could be achieved through cation exchange. To this end, a 1-butanol solution containing cetyltrimethylammonium bromide (CTAB) was layered on top of an aqueous solution of ONaRhMOP, and the resulting biphasic system was shaken for $5 \mathrm{~s}$ (Figure 1b; SI, Video S1). UV-vis of the aqueous phase showed that 48 molar equiv of CTAB ensured the quantitative transfer of ONaRhMOP from the water to the 1-butanol (SI, Figures S13 and S14). Complete transfer was further corroborated by ICP-OES, which revealed the absence of Rh(II) ions in the aqueous phase (SI, Table S3). The Rh(II)-MOP that had entered the 1-butanol was precipitated

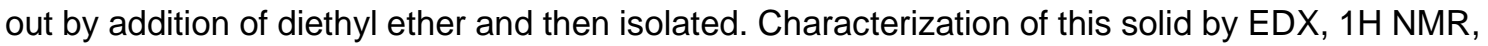
and ICP-OES (SI, Figures S15-S18 and Table S4) revealed formation of Rh(II)-MOP of formula $\mathrm{CTA}_{24}\left[\mathrm{Rh}_{2}(\mathrm{O}-\mathrm{bdc})_{2}\right]_{12}$ (hereafter referred to as OCTARhMOP), in which all the $\mathrm{Na}^{+}$cations of the previous $\mathrm{Rh}(\mathrm{II})-\mathrm{MOP}$ had been replaced by $\mathrm{CTA}^{+}$cations. These results confirmed that the phase transfer could be driven by cation exchange. Moreover, the stability of OCTARhMOP $24 \mathrm{~h}$ after the phase transfer in 1-butanol was confirmed through UV-vis (SI, Figure S19). Remarkably, replacing the $\mathrm{CTA}^{+}$cations with $\mathrm{Na}+$ cations enabled reverse transfer (Figure 1b): addition of 480 molar equiv of $\mathrm{NaCl}$ to the aqueous phase (10 times the concentration of CTAB) pushed the $\mathrm{Rh}$ (II)-MOP from the 1-butanol back into the water (Figure 1b; SI, video S1, Figures S20-S22).

Encouraged by our results with cation exchange-triggered phase transfer, we investigated whether this method could enable transfer of ONaRhMOP from water to less polar solvents, including aprotic organics solvents such as chloroform. To this end, an aqueous solution of ONaRhMOP was layered on top of a chloroform solution containing CTAB. Remarkably, the transfer from water to chloroform through the formation of OCTARhMOP (Figure 1b; SI, Figure S23) occurred spontaneously; indeed, shaking of the biphasic system was not even required. In this case, 96 CTAB molar equiv were necessary to complete the phase transfer, as confirmed by UV-vis, ICP-OES, EDX, and 1H NMR (SI, Figures S23-S28 and Tables S5 and S6). Again, the stability of OCTARhMOP chloroform solution $24 \mathrm{~h}$ after the phase transfer in chloroform was confirmed by UV-vis (SI, Figure S29). Furthermore, as addition of $\mathrm{NaCl}$ to the water phase did not enable the corresponding reverse transfer, we attempted to recover the parent OHRhMOP by protonating OCTARhMOP with TFA (Figure 1b). The $1 \mathrm{H}$ NMR spectrum of the precipitated solid after addition of TFA did not contain any peaks corresponding to CTA+ counter-cations, thereby confirming recovery of the initial OHRhMOP, which could be redissolved in 1-butanol (SI, Figure S30).

\section{Coupling the Two Phase Transfers: A Triphasic Solvent System}

Unlike the spontaneous phase transfer of OHRhMOP between water and chloroform that is triggered by cation exchange, the phase transfer of OHRhMOP between 1-butanol and water triggered by $\mathrm{pH}$ changes requires shaking. Therefore, to build an autonomous system in which Rh(II)-MOP could be spontaneously transferred through immiscible liquid phases solely via external chemical stimuli, we investigated the possibility of sequentially coupling the two phase transfers into a single system. Accordingly, we prepared a triphasic solvent system (1butanol/water/chloroform), which we used to test whether OHRhMOP would spontaneously cross from the 1-butanol into the chloroform phase (via the aqueous phase). The system comprised three layers, listed here by increasing density: a 1-butanol solution of
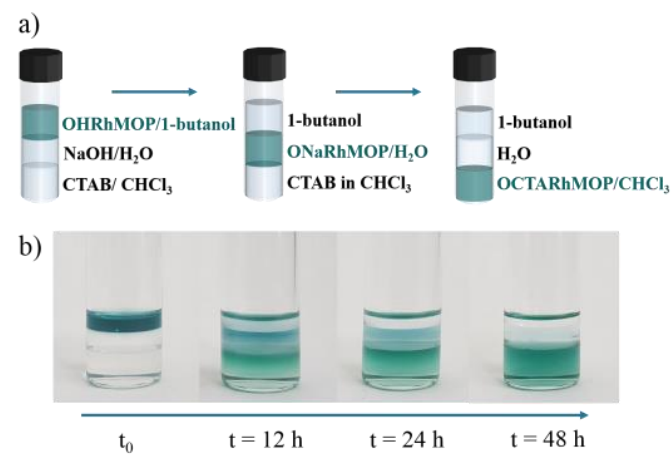

Figure 2. (a) Scheme showing the composition of the triphasic system and the subsequent phase transfers that spontaneously occurred. (b) Photographs showing the changes in the triphasic system over time 
OHRhMOP; an aqueous layer containing 24 molar equiv of $\mathrm{NaOH}$ and sodium chloride $(5 \%, \mathrm{w} / \mathrm{w})$; and a chloroform layer containing 96 molar equiv of CTAB (Figure 2). After $48 \mathrm{~h}, \mathrm{OHRhMOP}$ was found and characterized in the chloroform phase, confirming that combining the two phase transfers provides an autonomous system (SI, Figure S32). We reasoned that the spontaneous phase transfer between water and chloroform triggered by cation exchange drives the $\mathrm{pH}$ mediated phase transfer between water and 1-butanol, thus obviating the need for shaking.

\section{Cargo Transport}

We reasoned that, since the transfer of OHRhMOP between immiscible liquid phases requires only modification of its 24 surface hydroxyl groups, then the 12 exohedral axial sites of the Rh$\mathrm{Rh}$ paddlewheel clusters would remain accessible for functionalization via coordination chemistry.(21,23) Accordingly, we envisaged that said clusters could be used to bind cargo (via coordination bonds) to the surface of the OHRhMOP and that the resultant cargo-loaded OHRhMOP could then be transferred between phases. An example of possible cargo is $\mathrm{N}$-donor ligands, which strongly coordinate to such clusters and whose coordination can be readily followed, either by the naked eye or by monitoring spectroscopic changes of the band centered at $\lambda_{\max }=500-600 \mathrm{~nm}$, which corresponds to the $\pi^{*} \rightarrow \sigma^{*}$ transitions of the Rh-Rh bond. $(24,25)$

As proof-of-concept that OHRhMOP could deliver cargo via phase transfer, we chose 4aminopyridine (aminopyridine) as test cargo, considering that each OHRhMOP could coordinate 12 aminopyridine molecules to form the loaded product (hereafter called OHRhMOP(aminopyridine)). This loading ratio was confirmed by single-crystal X-ray diffraction (Figure 3a) and UV-vis ( $\lambda_{\max }$ shifted to $554 \mathrm{~nm}$; SI, Figure S33). As biphasic solvent systems, we chose water/chloroform and water/1-butanol, as aminopyridine is insoluble in chloroform and only slightly soluble in 1-butanol.

a)

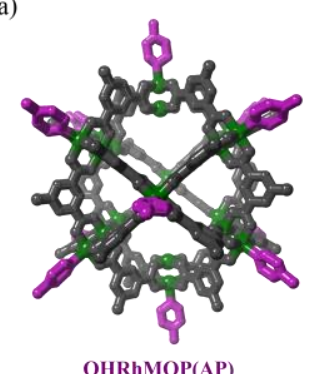

OHRhMOP(AP)

III

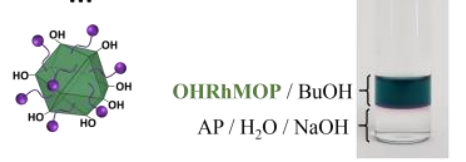

b) pH-triggered

$$
\text { pH-triggered }
$$

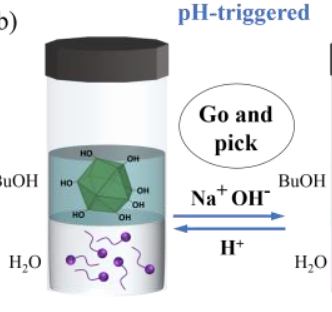

c)

c)

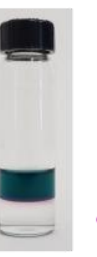

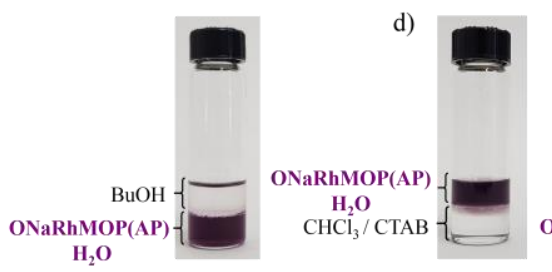

Cation Exchange-triggered
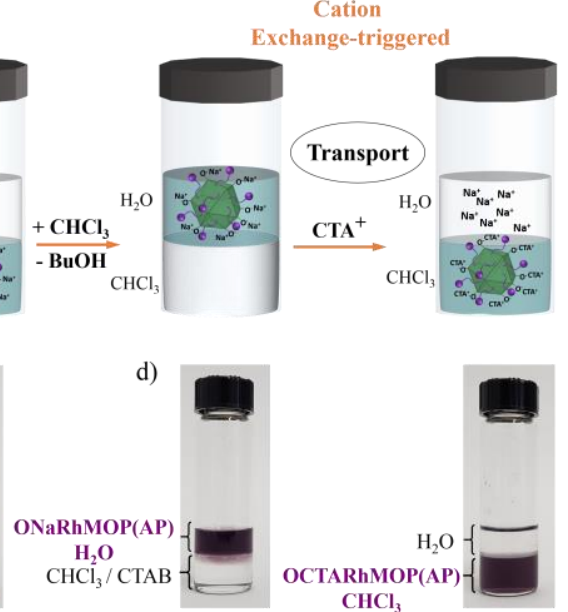

Figure 3. (a) Crystalline structure of OHRhMOP(aminopyridine). Color key: green, rhodium; gray, OH-bdc ligands; purple: aminopyridine molecules coordinated to the $\mathrm{Rh}$ (II) axial site. Hydrogen atoms and solvent molecules have been omitted for clarity. (b) Scheme representing loading of OHRhMOP with aminopyridine via $\mathrm{pH}$-driven phase transfer from 1-butanol to water driven by $\mathrm{pH}$ and subsequent cation exchange to trigger a second phase transfer, thus delivering the aminopyridine-loaded Rh-MOP into chloroform. (c) Photographs of the biphasic systems BuOH/OHRhMOP and aminopyridine/basic water before and after the phase transfers. (d) Photographs of the biphasic systems ONaRhMOP(aminopyridine)/water and $\mathrm{CHCl} / \mathrm{CTAB}$ before and after the phase transfers.

To test the transport of OHRhMOP(aminopyridine) from water to either chloroform or 1butanol, we first prepared an aqueous solution of ONaRhMOP containing 12 molar equiv of aminopyridine. Formation of ONaRhMOP(aminopyridine) was confirmed by UV-vis ( $\lambda_{\max }=555$ $\mathrm{nm}$ ) and 1H NMR (SI, Figures S34-S37). Note that ONaRhMOP(aminopyridine) could also be prepared from phase transfer of OHRhMOP in 1-butanol to a basic aqueous solution containing aminopyridine (Figure 3b; SI, Video S2). After formation of purple ONaRhMOP(aminopyridine), two biphasic systems were prepared: water/chloroform (containing 96 molar equiv of CTAB) and 
water/1-butanol (containing 48 molar equiv of CTAB). Complete transfer of ONaRhMOP(aminopyridine) to chloroform upon exchange of $\mathrm{Na}^{+}$ions with $\mathrm{CTA}^{+}$ions occurred spontaneously (Figure 2b; SI, Figure S40), whereas in 1-butanol, complete transfer required that the system be shaken for $5 \mathrm{~s}$ (SI, Figure S38 and Video S2). In each case, transfer was further confirmed by UV-vis and 1H NMR spectra (SI, Figures S38-S41).

We next explored cargo transfer in the opposite direction, from 1-butanol to water, using 4pyridinylboronic acid (PBA), an N-donor ligand that is insoluble in water, as cargo. Thus, a 1butanol solution of OHRhMOP was treated with 12 molar equiv of PBA, and the solution rapidly changed from green to purple. These spectroscopic changes were followed by UV-vis, which revealed a shift in $\lambda_{\max }$, from 596 to $554 \mathrm{~nm}$ (SI, Figure S42), which evidenced coordination of OHRhMOP to 12 molecules of PBA. A biphasic system was then prepared with the 1-butanol solution on top of a basic water layer. After shaking for $5 \mathrm{~s}$, transfer of OHRhMOP(PBA) to the aqueous phase by deprotonation of the Rh(II)-MOP was complete, as confirmed by UV-vis and $1 \mathrm{H}$ NMR (SI, Figures S43-S46).

Finally, we explored the amenability of OHRhMOP to transport larger molecules into solvents in which they are not soluble. To this end, we chose as cargo neutral red (NR), a phenazine-based dye with available $\mathrm{N}$-donor sites capable of coordinating to the axial sites of the Rh-Rh paddlewheels (SI, Scheme $\mathrm{S} 1)$. Since NR is soluble in 1-butanol but not in basic water or chloroform, we investigated its transport by two consecutive phase transfers: first, from 1-butanol to basic water and, second, from basic water to chloroform. The maximum number of NR molecules that OHRhMOP could transport to the basic aqueous phase was assessed (Figure 4a). Thus, solutions of OHRhMOP in 1-butanol were separately treated with different amounts of NR (from 1 molar equiv to 12 molar equiv). The resultant solutions were independently layered on top of basic water, the resulting biphasic systems were shaken for $5 \mathrm{~s}$, and finally, the fraction of NR that had been transferred into each aqueous phase was quantified by UV-vis of the 1-butanol phase (SI, Table S7). As depicted in Figure $4 \mathrm{~b}$, each OHRhMOP could transport up to four NR molecules into the aqueous phase. Furthermore, UV-vis analysis of the aqueous phases revealed that

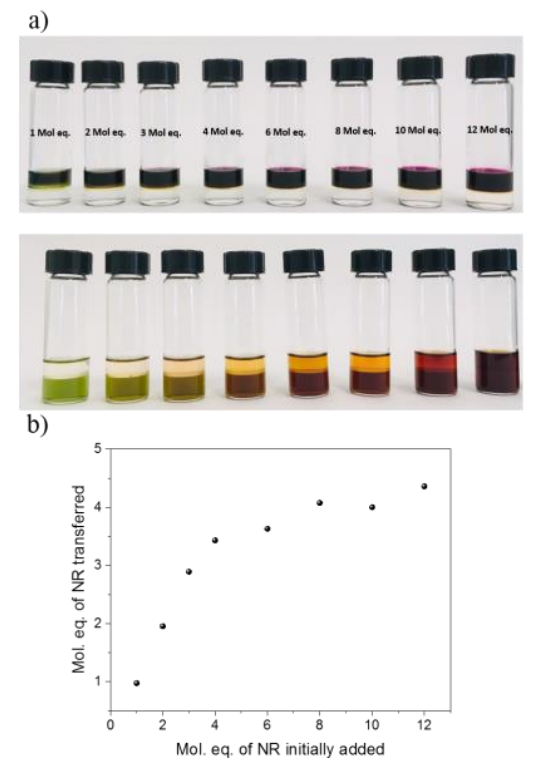

Figure 4. (a) Photographs of the biphasic systems containing OHRhMOP and NR in 1-butanol and basic water before (top) and after (bottom) shaking. (b) Amount of NR (in molar equiv) transferred in each experiment.

the molecular transport is driven by coordination of NR to Rh(II)-MOP (SI, Figure S49). Next, the aqueous phase containing the ONaRhMOP(NR) complex was layered on top of a chloroform phase containing CTAB. The ONaRhMOP(NR) was spontaneously and quantitatively transferred into the chloroform phase, having been mediated by cation exchange (SI, Figure S50).

\section{Role of Rh(II)-MOP in Cargo Transport}

Since, in our approach, the transport of cargo requires its coordinative anchoring to the outer $\mathrm{Rh}$ (II) sites of the paddle-wheels, we sought to investigate whether the Rh(II)-MOP would offer any advantage over the analogous paddle-wheel $\mathrm{Rh}(\mathrm{II})$ complex that constitutes the MOP scaffold. Accordingly, we synthesized the neutral $\mathrm{Rh}$ (II) paddle-wheel [Rh" ${ }_{2}$ (4-hydroxybenzoate) 4 ] by adapting a literature procedure(26) (SI, Figure S51) and then investigated its phase-transfer performance. Initially, we found that this Rh(II) dimer was insoluble in 1-butanol, thus preventing the $\mathrm{pH}$-triggered phase transfer between 1-butanol and water. We attributed the high solubility of OHRhMOP in various long-chain aliphatic alcohols-including in 1-octanol-to the higher density of hydroxyl groups (up to 24 per MOP) at its outer surface. 
Next, we investigated the phase transfer of $\left[\mathrm{Rh}_{2}{ }_{2}(4-\text { hydroxybenzoate) })_{4}\right]$ from water to chloroform via cation exchange. First, we dissolved the dimeric cluster in water by adding a stoichiometric amount of $\mathrm{NaOH}$ to deprotonate its four hydroxyl groups, which provided the corresponding sodium salt. However, all attempts to transfer this sodium complex from water to chloroform by exchanging $\mathrm{Na}^{+}$with $\mathrm{CTA}^{+}$failed (SI, Figure S53). Although spectroscopic characterization demonstrated that the $\mathrm{Na}^{+}$counterions of the $\mathrm{Rh}$ (II) complex had been replaced by $\mathrm{CTA}^{+}$, the transfer did not occur in less polar solvents (e.g., chloroform). In fact, in cases of poor solubility, a green solid precipitated out into the interphase (SI, Figures S53 and S54). On the basis of these results, we concluded that the paddle-wheel-based $\mathrm{Rh}$ (II) dimer does not undergo the same phase transfers that we had observed for OHRhMOP. This finding demonstrated the importance of concentrating several negative charges (in this case, 24 per MOP unit) or hydrophobic moieties at the MOP periphery to facilitate phase transfer-and by extension, cargo transport-between immiscible solvents.

\section{Molecular Separation through Phase Transfer of Rh(II)-MOPs}

Having transported diverse molecules between immiscible liquids by combining the coordination capability of $\mathrm{Rh}$ (II)-MOP with the phase transfers, we envisaged that selective coordination chemistry of $\mathrm{Rh}$ (II) centers could be exploited to separate physicochemically similar molecules that differ only in their affinity toward $\mathrm{Rh}$ (II) centers. We considered separation of a cyclic aliphatic compound from its corresponding aromatic counterpart, which is typically challenging due to their physicochemical similarity. $(27,28)$ Specifically, we postulated that the different basicity of aliphatic and aromatic heterocycles could be exploited to separate them via phase transfers of OHRhMOP.

As proof-of-concept for separation by phase transfer using Rh(II)-MOP, we chose the pair tetrahydrothiophene and thiophene (Figure 5). Note that, although we have previously reported examples of $\mathrm{N}$-donor cargo, $\mathrm{Rh}$ (II) ions also show excellent affinity toward S-donor ligands.(29) Thus, we exploited the difference in reactivity between the sulfur atom in tetrahydrothiophene, which serves as a basic site, and that in thiophene, whose electron pairs are significantly delocalized in the $\pi$ ring system, thus precluding any bonding to the Rh(II) acidic site (SI, Figure S55). We prepared a 1-butanol solution of OHRhMOP (1 mol equiv), thiophene (12 mol equiv),

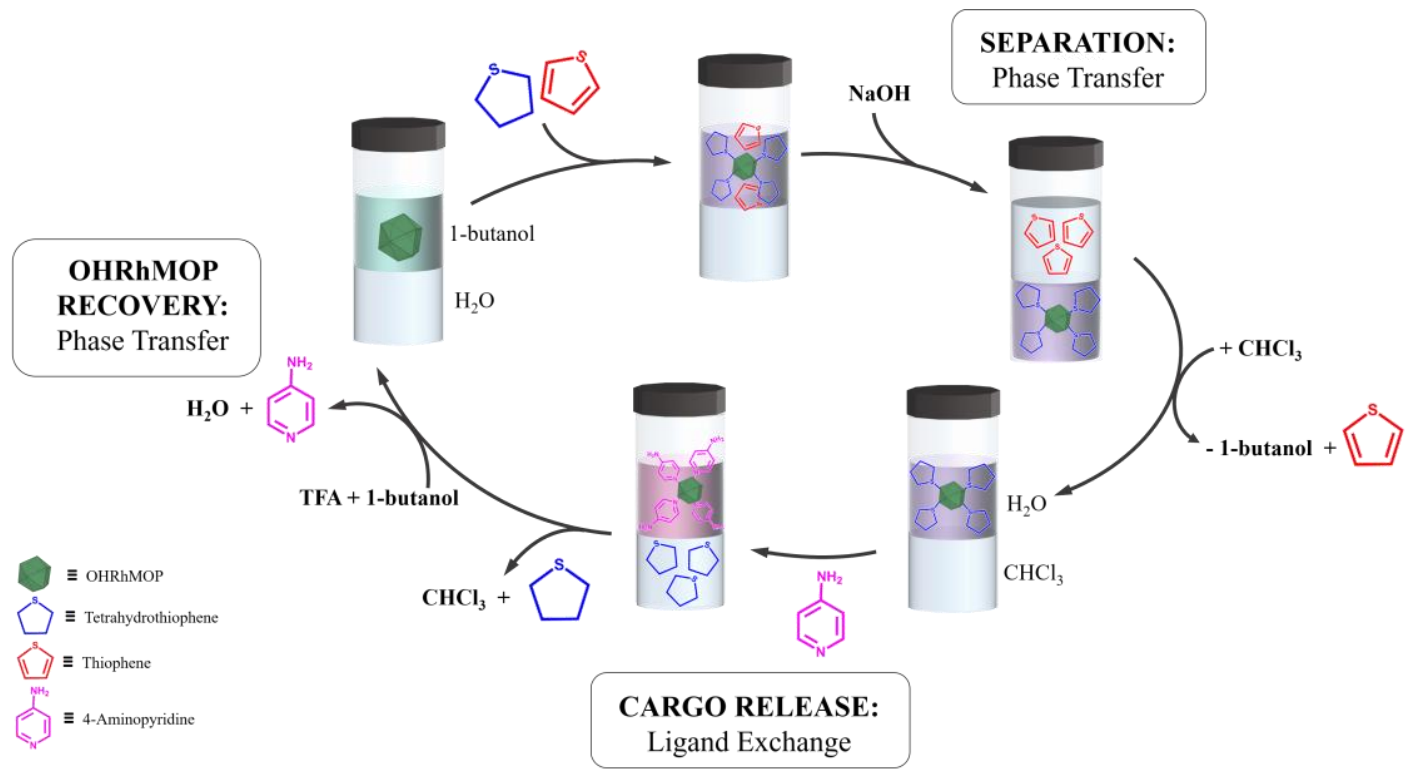

Figure 5. Scheme illustrating the application of phase transfer of Rh(II)-MOPs for molecular separation. Selective coordination of $\mathrm{Rh}(\mathrm{II})$-MOP to tetrahydrothiophene in 1-butanol and further phase transfer to the aqueous phase enable the separation. A subsequent ligand-exchange reaction with aminopyridine triggers the release of tetrahydrothiophene, which is recovered in the $\mathrm{CHCl}_{3}$ phase. Finally, a second phase transfer, triggered by addition of TFA, regenerates the MOP by transferring it to the 1-butanol phase and releasing the aminopyridine into the aqueous layer. 
and tetrahydrothiophene (12 molar equiv). Note that these compounds are soluble in 1-butanol but not in water. This solution was then layered on top of an aqueous solution of $\mathrm{NaOH}$ (24 molar equiv). After shaking for $5 \mathrm{~s}$, the OHRhMOP and tetrahydrothiophene transferred into the aqueous phase, whereas the (noncoordinating) thiophene remained in the 1-butanol layer. These results were supported by UV-vis and 1H NMR (SI, Figures S58 and S59).

We then explored release of the tetrahydrothiophene through ligand exchange based on the differential affinity of $\mathrm{Rh}(\mathrm{II})$ sites toward different functional groups. Given that $\mathrm{Rh}(\mathrm{II})$ ions preferentially bond to pyridines over thioethers,(29) we targeted the exchange of the coordinated tetrahydrothiophene with aminopyridine. Hence, 12 molar equiv of aminopyridine were added to the aqueous solution containing ONaRhMOP-tetrahydrothiophene, leading to a rapid change in color from violet to pink. UV analysis of the aqueous phase before and after the addition of aminopyridine revealed a shift in $\lambda_{\max }(565 \mathrm{~nm} \rightarrow 555 \mathrm{~nm})$, which confirmed the coordination of 12 molecules of aminopyridine to ONaRhMOP (SI, Figure S60). $1 \mathrm{H}$ NMR of the aqueous phase further confirmed the formation of ONaRhMOP-aminopyridine (SI, Figure S61). Consequently, tetrahydrothiophene was released and extracted into a $\mathrm{CDCl} 3$ layer, whose $1 \mathrm{H}$ NMR spectrum indicated pure tetrahydrothiophene without any trace of thiophene (SI, Figure S62). Note that without the use of aminopyridine, addition of chloroform to the ONaRhMOP-tetrahydrothiophene aqueous solution did not trigger tetrahydrothiophene release, further corroborating the requirement for ligand exchange (SI, Figure S63).

Finally, we explored recovery of the original OHRhMOP from the ONaRhMOP-aminopyridine aqueous solution via the aforementioned $\mathrm{pH}$-mediated phase transfer. We prepared a biphasic system with the aqueous solution and 1-butanol containing TFA. After shaking for $5 \mathrm{~s}$, the protonated OHRhMOP was found and characterized in the 1-butanol phase, whereas the released aminopyridine remained in the aqueous phase (SI, Figure S64).

\section{Conclusions}

We have demonstrated phase transfer of Rh(II)-MOPs between water and organic phases by $\mathrm{pH}$ changes and by cation exchange. Although protonation/deprotonation enables complete and reversible phase transfer from 1-butanol to water, exchange between hydrophilic and hydrophobic counter-cations can drive the opposite phase transfer, from water back into 1-butanol (or into chloroform). Moreover, we have designed a triphasic solvent system in which the $\mathrm{pH}$-changetriggered and cation-exchange-triggered phase transfers were sequentially coupled to yield an autonomous transfer mechanism. A unique feature of our phase transfer system vehicle is that it combines the functional groups of the organic linkers (responsible for shuttling the Rh(II)-MOP across immiscible liquids) and available axial sites of the $\mathrm{Rh}$ (II) paddle-wheels (reactive toward ligand exchange reactions) in a way that their relative position and stoichiometry are precisely known. Thus, the surface of the OHRhMOP shows two functionalities that present orthogonal reactivity and that can be used for different purposes and synergistically combined. This feature enables combining the phase transfer behavior of Rh(II)-MOP with its ability to coordinate up to 12 anchored molecules as cargo, thus enabling shuttling of diverse $\mathrm{N}$-donor ligands between immiscible liquids, including into solvents in which the cargo is insoluble. Moreover, we confirmed that bulky cargo could be delivered from 1-butanol to basic water to chloroform, through two sequential phase transfers. Such a mechanism of molecular transport is not based on host-guest encapsulation but on coordination chemistry, opening novel possibilities in the field of molecular separation. To demonstrate use of our approach in chemical separation based on selective coordination, we exploited the coordination abilities of Rh(II)-MOP to separate an aliphatic heterocycle (tetrahydrothiophene) from its aromatic analogue (thiophene). We showed that the coordination hierarchy of $\mathrm{Rh}$ (II) sites enables the release of tetrahydrothiophene through ligand exchange with aminopyridine and that OHRhMOP can be regenerated after the separation and release processes. We are confident that the possibility of transporting reagents into environments in which they are not naturally soluble, for their subsequent release via external chemical stimuli, will prove utile in diverse practical applications such as chemical separation, pollutant removal, and catalysis. 


\section{Materials and Methods}

Rhodium acetate, 1,3-benzenedicarboxylic acid, 5-hydroxy-1,3-benzenedicarboxylic acid, sodium hydroxide, tetrabutylammonium bromide (TBAB), benzyltributylammonium bromide (BTBAB), dodecyltrimethylammonium bromide (DTAB), cetyltrimethylammonium bromide (CTAB), dioctadecyldimethylammonium bromide (DODAB), tetraoctylammonium bromide (TOAB), 4-aminopyridine, 4-pyridinylboronic acid, trifluoroacetic acid, deuterium chloride, sodium chloride, neutral red, 4-hydroxylbenzoic acid, thiophene, and tetrahydrothiophene were purchased from Sigma-Aldrich and used as received. Solvents at HPLC grade were purchased from Fisher Chemicals.

UV-vis spectra were measured in an Agilent Cary 4000 at room temperature (ca. $25^{\circ} \mathrm{C}$ ). $1 \mathrm{H} \mathrm{NMR}$ spectra were acquired in a Bruker Avance III 400SB NMR spectrometer at "Servei d'Análisi Química" from the Autonomous University of Barcelona (UAB). The digested solutions were prepared following the same procedure. Roughly $10 \mathrm{mg}$ of sample was dissolved in a mixture of $500 \mu \mathrm{L}$ of DMSO-d6 and $20 \mu \mathrm{L}$ of DCl. The mixture was heated to $100{ }^{\circ} \mathrm{C}$ for $8 \mathrm{~h}$. Scanning electron microscopy (SEM)-EDX was performed in a SEM Quanta 650 FEM. ICP-OES was performed in a PerkinElmer Optima 4300DV at "Servei d'Análisi Química" from UAB. Mass spectroscopy (MALDI-TOF) measurements were performed in a 4800 Plus MALDI TOF/TOF (ABSCIEX, 2010). The matrix used in each case was trans-2-[3-(4-tert-butylphenyl)-2-methyl-2propenylidene]malononitrile for neutral $\mathrm{Rh}(\mathrm{II})$-MOPs and sinapinic acid for anionic Rh-MOPs. Neutral Rh(II)-MOPs were measured in positive mode, and anionic Rh(II)-MOPs were measured in negative mode. DLS and Z-potential data were acquired in a Zetasizer Nano ZS.

The Supporting Information is available free of charge on the ACS Publications website at DOI: 10.1021/jacs.9b10403.

Single-crystal X-ray diffraction of OHRhMOP(aminopyridine) (CCDC 1914907) and details of the syntheses and the phase transfer procedures; 1NMR, UV-vis, ICP-OES, MALDI-TOF, DLS, Zpotential, and EDX data (PDF)

Supporting movie (AVI)

Supporting movie (AVI)

X-ray crystallographic data (CIF) pdf: ja9b10403_si_001.pdf (13.26 MB)

microsoft video (avi) ja9b10403_si_002.avi (5.87 MB), ja9b10403_si_003.avi (4.48 MB)

crystallographic information file ja9b10403_si_004.cif (7.83 MB)

\section{References}

1. Yang, J.; Lee, J. Y.; Ying, J. Y. Phase Transfer and Its Applications in Nanotechnology. Chem. Soc. Rev. 2011, 40, 1672

2. Michalet, X. Quantum Dots for Live Cells, in Vivo Imaging, and Diagnostics. Science 2005, 307, 538

3. Shirasaki, Y.; Supran, G. J.; Bawendi, M. G.; Bulović, V. Emergence of Colloidal Quantum-Dot Light-Emitting Technologies. Nat. Photonics 2013, 7, 13

4. Imura, Y.; Morita, C.; Endo, H.; Kondo, T.; Kawai, T. Reversible Phase Transfer and Fractionation of Au Nanoparticles by pH Change. Chem. Commun. 2010, 46, 9206

5. Peng, L.; You, M.; Wu, C.; Han, D.; Öçsoy, I.; Chen, T.; Chen, Z.; Tan, W. Reversible Phase Transfer of Nanoparticles Based on Photoswitchable Host-Guest Chemistry. ACS Nano 2014, 8, 2555 
6. Edwards, E. W.; Chanana, M.; Wang, D.; Möhwald, H. Stimuli-Responsive Reversible Transport of Nanoparticles Across Water/Oil Interfaces. Angew. Chem., Int. Ed. 2008, 47, 320

7. Feng, X.; Ma, H.; Huang, S.; Pan, W.; Zhang, X.; Tian, F.; Gao, C.; Cheng, Y.; Luo, J. Aqueous-Organic Phase-Transfer of Highly Stable Gold, Silver, and Platinum Nanoparticles and New Route for Fabrication of Gold Nanofilms at the Oil/Water Interface and on Solid Supports. J. Phys. Chem. B 2006, 110, 12311

8. Liu, J.; Alvarez, J.; Ong, W.; Román, E.; Kaifer, A. E. Phase Transfer of Hydrophilic, Cyclodextrin-Modified Gold Nanoparticles to Chloroform Solutions. J. Am. Chem. Soc. 2001, 123, 11148

9. Gittins, D. I.; Caruso, F. Spontaneous Phase Transfer of Nanoparticulate Metals from Organic to Aqueous Media. Angew. Chem., Int. Ed. 2001, 40, 3001

10. Grommet, A. B.; Hoffman, J. B.; Percástegui, E. G.; Mosquera, J.; Howe, D. J.; Bolliger, J. L.; Nitschke, J. R. Anion Exchange Drives Reversible Phase Transfer of Coordination Cages and Their Cargo. J. Am. Chem. Soc. 2018, 140, 14770

11. Grommet, A. B.; Nitschke, J. R. Directed Phase Transfer of an Fell4 L4 Cage and Encapsulated Cargo. J. Am. Chem. Soc. 2017, 139, 2176

12. Zhang, S.-Y.; Kochovski, Z.; Lee, H.-C.; Lu, Y.; Zhang, H.; Zhang, J.; Sun, J.-K.; Yuan, J. Ionic Organic Cage-Encapsulating Phase-Transferable Metal Clusters. Chem. Sci. 2019, 10, 1450

13. Eddaoudi, M.; Kim, J.; Wachter, J. B.; Chae, H. K.; O'Keeffe, M.; Yaghi, O. M. Porous Metal-Organic Polyhedra: $25 \AA$ Cuboctahedron Constructed from 12 Cu2(CO2)4 PaddleWheel Building Blocks. J. Am. Chem. Soc. 2001, 123, 4368

14. Li, J.-R.; Zhou, H.-C. Bridging-Ligand-Substitution Strategy for the Preparation of Metalorganic Polyhedra. Nat. Chem. 2010, 2, 893

15. Li, J.-R.; Yu, J.; Lu, W.; Sun, L.-B.; Sculley, J.; Balbuena, P. B.; Zhou, H.-C. Porous Materials with Pre-Designed Single-Molecule Traps for CO2 Selective Adsorption. Nat. Commun. 2013, 4, 1538

16. Teo, J. M.; Coghlan, C. J.; Evans, J. D.; Tsivion, E.; Head-Gordon, M.; Sumby, C. J.; Doonan, C. J. Hetero-Bimetallic Metal-organic Polyhedra. Chem. Commun. 2016, 52, 276

17. Park, J.; Perry, Z.; Chen, Y.-P.; Bae, J.; Zhou, H.-C. Chromium(II) Metal-Organic Polyhedra as Highly Porous Materials. ACS Appl. Mater. Interfaces 2017, 9, 28064

18. Liu, G.; Di Yuan, Y.; Wang, J.; Cheng, Y.; Peh, S. B.; Wang, Y.; Qian, Y.; Dong, J.; Yuan, D.; Zhao, D. Process-Tracing Study on the Postassembly Modification of Highly Stable Zirconium Metal-Organic Cages. J. Am. Chem. Soc. 2018, 140, 6231

19. Rowland, C. A.; Lorzing, G. R.; Gosselin, E. J.; Trump, B. A.; Yap, G. P. A.; Brown, C. M.; Bloch, E. D. Methane Storage in Paddlewheel-Based Porous Coordination Cages. J. Am. Chem. Soc. 2018, 140, 11153

20. Carné-Sánchez, A.; Albalad, J.; Grancha, T.; Imaz, I.; Juanhuix, J.; Larpent, P.; Furukawa, S.; Maspoch, D. Postsynthetic Covalent and Coordination Functionalization of Rhodium(II)-Based Metal-Organic Polyhedra. J. Am. Chem. Soc. 2019, 141, 4094

21. Carné-Sánchez, A.; Craig, G. A.; Larpent, P.; Guillerm, V.; Urayama, K.; Maspoch, D.; Furukawa, S. A Coordinative Solubilizer Method to Fabricate Soft Porous Materials from Insoluble Metal-Organic Polyhedra. Angew. Chem., Int. Ed. 2019, 58, 6347

22. Carné-Sánchez, A.; Craig, G. A.; Larpent, P.; Hirose, T.; Higuchi, M.; Kitagawa, S.; Matsuda, K.; Urayama, K.; Furukawa, S. Self-Assembly of Metal-organic Polyhedra into Supramolecular Polymers with Intrinsic Microporosity. Nat. Commun. 2018, 9, 2506

23. Furukawa, S.; Horike, N.; Kondo, M.; Hijikata, Y.; Carné-Sánchez, A.; Larpent, P.; Louvain, N.; Diring, S.; Sato, H.; Matsuda, R.; Kawano, R.; Kitagawa, S. RhodiumOrganic Cuboctahedra as Porous Solids with Strong Binding Sites. Inorg. Chem. 2016, 55,10843

24. Warzecha, E.; Berto, T. C.; Berry, J. F. Axial Ligand Coordination to the C-H Amination Catalyst Rh2(Esp)2: A Structural and Spectroscopic Study. Inorg. Chem. 2015, 54, 8817 
25. Dennis, A. M.; Howard, R. A.; Bear, J. L. The Reactivity of Tetra- $\mu$-acetatodirhodium(II) with Selected Di- and Tripeptides, Substituted Pyridines and Imidazole Ligands. Inorg. Chim. Acta 1982, 66, 31

26. Sveshnikov, N. N.; Dickman, M. H.; Pope, M. T. Bis(dimethyl sulfoxide-S)tetrakis( $\mu$-phydroxybenzoato-O:O')dirhodium(II)-tetrakis(I-butyrato-O:O')bis(dimethylsulfoxide-

S)dirhodium(II) cocrystal ethanol disolvate. Acta Crystallogr., Sect. C: Cryst. Struct. Commun. 2000, 56, 1193

27. Garcia-Villaluenga, J. P.; Tabe-Mohammadi, A. A review on the separation of benzene/cyclohexane mixtures by pervaporation processes. J. Membr. Sci. 2000, 169, 159

28. Jie, K.; Zhou, Y.; Li, E.; Zhao, R.; Huang, F. Separation of Aromatics/Cyclic Aliphatics by Nonporous Adaptive Pillarene Crystals. Angew. Chem., Int. Ed. 2018, 57, 12845

29. Kitchens, J.; Bear, J. L. A Study of some Rhodium(II) Acetate Adducts. J. Inorg. Nucl. Chem. 1969, 31, 2415 